\title{
Cuestión de género: algunos aspectos clave del feminismo en la creación artística posmoderna
}

\author{
Luz del Carmen Magaña Villaseñor ${ }^{1}$
}

Recepción: 1 de abril de 2014 / Aprobación: 18 de mayo de 2015

\section{Resumen}

El presente trabajo se enfocará, principalmente, en la segunda mitad del siglo $X X$, partiendo del movimiento posmoderno buscará plantear el momento específico donde empezó el acto de acción feminista y por quiénes fue liderado, qué expectativas o beneficios alcanzaron las primeras feministas, cuál fue el motivo primordial para que las mujeres se levantaran a exigir igualdad al lado del hombre. Esta es una investigación de los movimientos feministas dentro del arte del performance así como de las reivindicaciones de mujeres por medio de este, de conflictos con su entorno social tanto como con el género masculino con el fin de hacer una comparación entre estas mujeres -artistas reivindicativas de la historia conocida y no conocida de María Magdalena- y cómo por medio de sus reivindicaciones, también se limpia y purifica el estigma de la Magdalena bíblica, "la prostituta".

\section{Palabras clave}

Arte; feminismo; performance; posmoderno; reivindicación

\section{Abstract}

Was focusing principally in the second half of the 20th century, departing from the postmodern movement, to raise the specific moment, and where feminist began the act of action and by whom it was led, what expectations or benefits the first feminists reached, and which was the basic motive in order that the women were getting up to demanding an equality next to the man. It is an investigation of the movements feminists inside the art of the performance, and the women's recoveries by means of this one, of conflicts with his social environment and with the masculine kind; for this way, protest artists do a comparison or similarity between these women-, and the known history, and not known about Maria Magdalena, and as by means of his recoveries, also there is cleaned and purifies the stigma of the Biblical Sponge-cake, "the prostitute".

1 Mexicana. Maestra en Artes Visuales con énfasis en Pintura por la Academia de San Carlos de la Universidad Nacional Autónoma de México. Doctoranda en Artes en la Universidad de Guanajuato y Catedrática de Arte en la Universidad Autónoma de Querétaro, México. Artista Visual y performancera con exposiciones individuales y colectivas nacionales e internacionales. Contacto al correo electrónico: luzdelcarmenmagana@hotmail.com o en la página http://www.luzdelcarmenmagana.com 


\section{Key words}

Art; feminist; performance; postmodern; recovery

\section{Resumo}

Eles se concentram principalmente na segunda metade do século XX, com base no movimento pós-moderno, elevar o tempo especificado, e onde o ato de ação feminista começou e que foi levado, o que as expectativas ou benefícios alcançados feminista cedo, e que foi a principal razão para que as mulheres se levantam para exigir a igualdade ao lado do homem. É uma investigação dos movimentos feministas na arte da performance, e reivindicações das mulheres por meio deste, os conflitos com seu ambiente social e com o gênero masculino assim, fazer uma comparação ou semelhança entre essas mulheres-artistas de protesto e história conhecidos e desconhecidos de Maria Madalena, e também através de suas reivindicações, também limpa e purifica o estigma da Madalena bíblica, "a prostituta".

\section{Palavras chave}

Arte; feminista; o desempenho; pós-moderna; a reivindicação

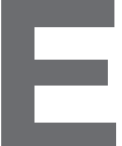

n la segunda década del siglo XX se podía encontrar, de manera frecuente, a las mujeres ocupando alrededor del mundo, las posiciones asignadas a los hombres, por ejemplo, dentro de la ciencia, la medicina o la industria. Estos casos fueron, en principio, considerados tabú, dado que era inconcebible que una mujer llegara a asumir los trabajos que estuvieran fuera del hogar. Lo anterior provocó que la mujer, poco a poco, se hiciera presente dentro del marco de la historia. La condición de la mujer occidental, blanca, de clase media ha mejorado sustancialmente y, lo cierto es que, movimientos como el feminismo, la independencia, el voto y la libertad sexual han secundado para que esto sea factible.

En este momento, las mujeres tienen que lidiar con diferentes factores para sobresalir y poder demostrar sus capacidades dentro de la comunidad donde se desenvuelven. Ellas viven en una sociedad dominada no solo por un patriarcado, sino cada vez más, y de una manera sumamente provocadora, por el culto a la belleza y la estética. En la actualidad, se busca la juventud eterna, donde las víctimas esenciales son las mujeres y su corporalidad. Las operaciones estéticas son cada vez más habituales en todas las franjas de edad, nivel social y económico en todo el planeta, lo cual ha acrecentado una visión femenina diferente en pleno siglo XXI.

El presente ensayo se enfoca en la segunda mitad del siglo XX, partiendo del movimiento posmoderno y de cómo se libró la batalla contra el machismo en los países desarrollados como Estados Unidos, particularmente, la del movimiento del performance. Busca responder las preguntas: ¿cuáles fueron las 
razones para que los movimientos de artistas feministas eligieran el performance (arte de acción) dentro de la posmodernidad para expresar sus inquietudes con lo que sucedía en su vida cotidiana? y ¿qué objetivos buscaban? Aunque estas interrogantes ya han sido abordadas y analizadas por varias teóricas de la historia del arte (Griselda Pollok y Amelia Jones), lo que atañe a esta investigación son los movimientos feministas dentro del arte del performance: la construcción de la mujer como sujeto, los feminismos de la igualdad, los feminismos de la identidad y las diferentes reivindicaciones de mujeres por medio de estos movimientos. Tomando la idea de reivindicación como la inclusión del Otro por medio de la diferencia, para así ocupar el lugar antes arrebatado dentro de su entorno y su historia, en la cual es evidente la exclusión de las mujeres dentro del contexto artístico.

Sería una lástima terrible, que las mujeres escribieran como los hombres, o se parecieran físicamente a los hombres, porque dos sexos son ya pocos, dada la vastedad y variedad del mundo; ¿cómo nos las arreglaríamos, pues, con uno solo? (Woolf, 1992, 192).

Al haber sido excluidas de la historia del arte, donde el único que figuraba era el hombre blanco, europeo, heterosexual y de clase media -como se ha planteado en distintos estudios críticos-, se desarrolló el trabajo de las primeras artistas feministas, durante las décadas de 1950 y 1960, tomando el performance como el mayor representante artístico y el cuerpo como el soporte principal por excelencia.

Las feministas hicieron un planteamiento de los conflictos sobre su entorno social y el género masculino; para así, establecer una comparación entre estas mujeres artistas reivindicadoras dentro del mundo del arte y la historia conocida de María Magdalena. Al hacer el abordaje por medio de sus reivindicaciones, también se limpia y purifica el estigma de la Magdalena bíblica, "la prostituta".

Un claro ejemplo de lo anterior, es la célebre protesta contra la exposición Panorama internacional de la pintura y la escultura recientes, para inaugurar las nuevas salas del Museum of Modern Art de Nueva York, en 1984. Propuesta que reunía a 165 artistas, de los cuales solo 14 eran mujeres.

La década de 1970 representó el desarrollo de un pluralismo y una ampliación de los horizontes de las prácticas artísticas, aunque también se observa una oposición silenciosa a la participación de las mujeres y las minorías dentro de estas. Por entonces, algunas mujeres habían tratado de resolver su quehacer artístico enfrentándose contra la tradición existente, proponiendo nuevos temas y nuevos soportes artísticos. Como respuesta a la famosa pregunta: ¿por qué no ha habido grandes mujeres artistas?, Linda Nochlin plantea:

Es muy simple; estaban pariendo, estaban haciendo la comida, estaban fregando. Estaban facilitando el triunfo de sus parejas, en una demostración todavía no suficientemente estudiada de cómo el Síndrome de Estocolmo 
afecta especialmente a las mujeres, tradicionalmente esclavas del entorno afectivo y familiar (Nochlin citada en Aliaga, 2008, 37).

Ahora, en el siglo XXI, parece que el feminismo sigue siendo necesario, ya no de una manera tan radical, pero sigue estando muy presente dentro de la historia femenina del arte. Durante la década de 1970, las feministas radicales no aceptaban las acciones feministas de las mujeres que les parecían "bellas" por creer que la belleza les facilitaba algunos privilegios dentro de la sociedad masculina y hasta se cuestionó si una mujer bella podría ser feminista y más aún, artista, como en el caso de Carolee Schneemann o Hanna Wilke; artistas del performance que utilizaban su cuerpo en una serie de retratos en movimiento donde el estereotipo de la belleza se fusiona con el cuerpo como objeto femenino expuesto y acentuado por poses sensuales que cuestionan los prejuicios del hombre acerca de la forma de ver el cuerpo femenino. Dicho concepto no funciona de manera radical, pero esta forma de proceder, fue el detonante para que la mujer empezara a unirse y a trabajar en conjunto con otras mujeres accionistas, lo cual, convenientemente, sucedió de una manera más específica en el arte acción o performance; manifestación artística que, por mucho tiempo, llevó la bandera de movimiento de minorías.

El feminismo en el arte no ha limpiado la ideología que existe dentro del núcleo de este y sus mercados, la cual es que el género masculino lleva la batuta de la acción, aunque esto no es solo particular del arte, aunque sí ha ayudado y extendido el panorama de la mujer dentro de este buscando, por medio del arte, igualdad.

En la década de 1940, se logró progresivamente el voto de la mujer, a partir de entonces, en diversos países del mundo, llega un periodo de calma aparente. Sin embargo, en la década de 1960, se produce el renacer del movimiento en un contexto sociocultural de reivindicación contra el conformismo social y el sistema, el cual se afianzó en mayo de 1968, como producto de las protestas a causa de la Guerra de Vietnam y a favor de los derechos sociales, civiles y humanos dando origen al feminismo como se conoce actualmente.

A pesar de que la actividad a favor de los derechos de las mujeres tuvo un auge mayor durante la década de 1960, cuando a este movimiento se le denominó segunda ola, el feminismo como movimiento surgió en los primeros años de la posguerra, en 1950 cuando Eleonor Roosevelt y algunas representantes de los países del tercer mundo, todas ellas comprometidas activistas de la ONU, transformaron el término "derechos del hombre" por el de "derechos humanos", el cual incluía, por primera vez, al género femenino. Durante este tiempo, la obra El segundo sexo de Simone de Beauvoir se pone en discrepancia con el materialismo que no reconoce al género femenino como sujeto de la historia, sino como objeto de la misma y mostró ciertas fallas del psicoanálisis, debido a que algunas de sus premisas presentan al género masculino como lo único y universal, mientras que el femenino es simple expresión de la carencia de lo masculino. Este texto se convirtió en una referencia importante 
y fundamental al cuestionar la desigualdad de las mujeres en la época, cuando la igualdad era una realidad.

Durante los años sesenta todas las convenciones y los valores aprendidos como universales, incontrovertibles y eternos, comenzaron a cuestionarse y debatirse abiertamente. Frente a los conflictos bélicos de Corea y Vietnam se despertó un movimiento pacifista que acogió tanto a veteranos que deseaban eliminar la opresión, la injusticia y el racismo en Europa y en Japón, como a pacifistas que se organizaron para poner fin a las guerras, a la vez que se iniciaba un fuerte movimiento en pro del cese de la discriminación segregacionista y el ejercicio pleno de los derechos civiles para toda la población norteamericana (Alario Trigueros, 2005, 50).

A causa de estos hechos, muchas mujeres empezaron a preguntarse sobre el papel que jugaban en este proceso histórico. Yoko Ono y John Lennon escribieron después la canción Whoman is the nigger of the world, sencillo que salió en el año de 1972, canción que protesta contra el papel de inferioridad impuesto a la mujer dentro de la sociedad, esta canción hace énfasis en la frase: "las mujeres son los negros de la humanidad", colocándolas en el papel de renegadas dentro de un mundo cambiante y con esto mostraban la otra cara de las relaciones de género en la sociedad. Así, las mujeres empezaron a constituir agrupaciones reducidas de manera consciente formando los primeros grupos feministas contemporáneos, donde funge como principal aporte la equidad en el ejercicio de los Derechos Humanos.

Artistas como Marisol Escobar, Lee Kranser, Eva Hesse, Niki de SaintPhalle y Louise Bourgeois son la referencia al arte norteamericano feminista y a la primera generación de artistas creadoras de acciones y representaciones de la década de 1970; donde, primero en Estados Unidos y después en el resto de Occidente, se desarrolló un movimiento feminista dentro de las artes visuales, en especial, las artes plásticas, cuestionando lo que, hasta ese momento, se consideraba obra de arte.

En la posmodernidad, la teoría y la practica feminista dentro del arte investiga nuevas propuestas que mantienen una conexión entre las mujeres dentro del movimiento feminista y las mujeres artistas que, en ese tiempo, utilizaron su propio cuerpo como fuente inagotable de simbolismos y metáforas para crear su obra.

El movimiento feminista en las artes -es decir, el compromiso de lograr un arte que refleje la conciencia política y social de las mujeres- transformó la práctica del arte en Norteamérica durante este período poniendo constantemente en tela de juicio y desafiando los supuestos e ideologías patriarcales del arte y artista (Chadwick, 1992,78).

Por esta razón, una de las primeras acciones reivindicadoras de las mujeres artistas feministas dentro del mundo de las artes visuales se realizó en 1969, en una exposición colectiva que se celebraba cada año en Whitney Museum de Nueva York, en la cual un porcentaje muy pequeño que exponía era 
del género femenino, a causa de esto, un grupo de mujeres que pertenecían al gremio artístico protestaron a las puertas de este museo por medio de acciones espontáneas que, mediante diferentes descontextualizaciones, mostraban su inconformidad con este hecho, creando así el movimiento WAR (Women Artists in Resistence).

De igual manera, se llevó a cabo el primer curso de arte feminista, en Fresno, California en 1970, bajo la batuta de la escultora, pintora y después performancera Judy Chicago, artista radical feminista, probablemente una de las primeras mujeres en reivindicar la historia del género femenino dentro del arte y, quien después de su divorcio, se despojó de su apellido de casada para adjudicarse el de "Chicago" en un cuadrilátero de boxeo, por ser el nombre de la ciudad donde nació. Asimismo, se funda el Feminist Art Program, desarrollado en el Institute of Art de Los Ángeles, California en 1971, promovido por Judy Chicago y Miriam Shapiro, otra artista del performance, reivindicando las creaciones realizadas por mujeres dentro de la historia del arte.

Shapiro toma conciencia feminista y junto a Judy Chicago pone en marcha el Programa de Arte Feminista, en el California Institute of Arts, dirigido exclusivamente a mujeres y en el que formaron parte 22 alumnas en la primera edición, con quienes crean la primera exposición feminista en una casa abandonada en Nueva York con el título "Womanhouse". En palabras de la crítica Patricia Mayayo:

Como resultado del trabajo de este programa, en 1972 se organizó una muestra colectiva titulada: Womanhouse, en la que veintidós de las artistas participantes en el programa crearon obras, restaurando y ambientando una casa abandonada con objeto de tratar temas como la domesticidad, el cuerpo, la identidad o los estereotipos. En este proyecto participaron, además de Judy Chicago y Miriam Shapiro, Faith Wilding, Robin Mitchel, Karen Le Coq, Serry Brody, Susan Frazier, Vicki Hogetts, Robin Weltscher, Camile Gray, Robin Schiff, Beth Barchenmeier, Sandy Orgel, Nancy Youdelman y Kathy Huberland entre otras. La cual evocaba, con crudeza, el drama del ama de casa atrapada en la ética del sacrificio: los muros se hallaban cubiertos de una multitud de esculturas en forma de huevo frito, que se iban transformando paulatinamente en senos femeninos, una representación metafórica del papel nutricio protector atribuido tradicionalmente a las mujeres (Mayayo citada en Alario Trigueros, 2005, 53).

Sin embargo, el corolario de esa evaluación de arte femenino es colocarnos en el mismo centro del problema, tal como lo marcó el éxito creciente de mujeres artistas predominantemente blancas, de tendencia heterosexual y clase media como Barbara Kruger, Mary Kelly, Cindy Sherman y Cornelia Parker. Lo anterior tiene que ver con un interés renovado por vender una política de identidad, así como por agotar las existencias de la misma mediante etiquetas de post identidad; todo esto para lograr un encomiable deseo de incluir a las mujeres artistas en las historias del arte, tanto del arte moderno como del arte contemporáneo y así, considerar los gigantescos cambios mundiales para 
recuperar con más éxito los diferentes aspectos de un movimiento político y radical dentro del feminismo en la creciente historia del arte.

La práctica feminista dentro del performance consiste en que las estrategias deben ser, desde el punto de vista cultural, específicas según el contexto en que se apliquen, de manera metafórica, se habla desde el vacío del núcleo central de lo planteado en lugar de poner en escena la carne "presente" para ser capturada por la lente de la cámara en un desafortunado efecto fetichista. De manera paradójica, acepta la ausencia, el vacío situado en el centro de la existencia humana, con el fin de representar una actuación parafeminista politizada. Las prácticas feministas dentro del arte que más interesan en este momento sostienen el legado más importante del feminismo de las décadas de 1960 y 1970: la exposición insistente de los circuitos de poder a través de los cuales se identifican los sujetos y se sitúan en la cultura y/o la articulación gloriosa de cuerpos sexualizados en una gama de identificaciones complejas; cuerpos que, de un modo u otro, enuncian un tipo de actuación que les permite hablar contra la veta de discriminación racista, clasista, homofóbica y de otra naturaleza; todo lo cual es también inherentemente sexista y contrario a la mujer.

De ahí que el valor del arte feminista no está en el tiempo, ni en el contexto de este, sino, de modo espec, en lo que cada una de las mujeres, de manera individual y autónoma, quiere expresar y reivindicar de su entorno, de su género, de la vida en general y de su vida en particular, sabiendo que cada una como mujer tiene una historia diferente y que también existen minorías dentro de las minorías, como las mujeres negras, musulmanas, latinas, tomando en este momento el estandarte de Marías Magdalenas y reivindicando a la mujer, en general, por medio de su historia y de su contexto de vida, y con estas acciones performativas, reivindicando también el juicio que se le ha impuesto a la imagen iconográfica de María Magdalena a lo largo de los siglos donde es vista como una prostituta y una pecadora arrepentida.

Las mujeres dentro del feminismo, en la época de la posmodernidad, se encuentran con nuevas condiciones institucionales, sociales y políticas, lo cual marca un punto de transformación dentro de la lucha por hacer el trabajo de las mujeres visible, por establecer la oportunidad de la heterogénea participación de la producción artística. Es importante que las nuevas generaciones dentro de los movimientos feministas sean conscientes de su historia creadora, más que dominar una cronología de eventos pasados, se debe reconocer el valor de las distintas reivindicaciones que, por medio de su arte y de su cuerpo, han aportado a la historia del arte. Tanto en una reivindicación o afianzamiento de la identidad como en demostrar las estrategias para la creación de estos cambios y su representación.

En conclusión, el feminismo no ha logrado destruir las estructuras machistas que continúan sustentando el mundo del arte, lo que ha conseguido, en 
relación con las artes visuales, es una aceptación a gran escala de algo tan viable como lo masculino y lo femenino, es decir, donde puede haber cabida para los dos géneros por igual. El feminismo se debe adaptar a las identificaciones de nacionalidad, filiación religiosa, vínculos de parentesco y clases sociales que condicionan el trato de la mujer en cada caso específico. Se debe reconocer la imposibilidad de saber cuál es la categoría o la experiencia de la mujer a través de las fronteras, las épocas y los lugares, pero al mismo tiempo, se deben generar críticas absolutamente inflexibles a los abusos de la violencia misógina. Por ello se necesita una política renovada que conserve un compromiso sólido con lo que representa el feminismo, el reconocimiento de que la forma en que se identifican las personas en términos de género y sexualidad condicionan el modo en que se posicionan en los ámbitos social y personal. Y este papel también le corresponde al arte en general, aunque el performance ya ha tomado un lugar específico dentro de las reivindicaciones de la mujer, las que por medio de esta expresión artística se han liberado del yugo en que la sociedad masculina las ha puesto y ha puesto su obra artística.

Una nueva generación de artistas, en su mayoría mujeres, han hecho uso de la savia política que dota de poder al feminismo, apropiándose de algunas estrategias de previo efectivas y privándolas, en algunos casos, de su potencial para generar fricción en relación con las estructuras económicas e ideológicas que sustentan el valor de la obra de arte. Estas obras indican que, en lugar de cerrar el círculo, lo más importante es comprender qué es lo que implica el mantenimiento del vacío dentro de la historia del arte, mientras se insiste en la certidumbre de los momentos cruciales en contextos particulares, cuando se deben desafiar las desigualdades que dan como resultado la opresión, articulando la incertidumbre del vacío mediante un cuerpo concreto representado en un contexto institucional específico. De esta manera, nos damos cuenta de que el feminismo como tal sigue siendo un movimiento en progreso y, más aún, dentro de la historia del arte, y aunque se ha valido del performance para poder expresar muchos de sus argumentos, aún no sabemos cómo concluirá, mientras sigan existiendo minorías, no solamente de género, sino de raza y de sexualidades, los movimientos surgirán, y en ellos, siempre habrá mujeres artistas que irán creando y reivindicando desde su propio entorno y su propio cuerpo.

\section{Referencias}

Alario Trigueros, María Teresa. (2005). Arte y feminismo. Arte hoy. España: Editorial Nerea.

Alcázar, Josefina y Fuentes, Fernando. (2005). Performance y arte acción en América latina. México: Editorial Citru y Ediciones sin nombre. 
Aliaga, Juan Vicente. (2004). Arte hoy, arte y cuestión de género. La segunda oleada feminista en Estados Unidos. España: Editorial España.

Beauvoir, Simone. (2011). El segundo sexo. México: Editorial Debolsillo.

Chadwick, Whitney. (1992). Crítica feminista: el género como collage. España: Editorial España.

Jones, Amelia. (2011). El cuerpo del artista. Estados Unidos: Editorial Phaidon.

Eco, Umberto. (2007). Historia de la fealdad. México: Editorial Debolsillo.

Olivares, Rosa. (2001). Escritos sobre la piel. Revista Exit, (2).

Olivares, Rosa. (2006). Performance. Revista Exit, (42).

Olivares, Rosa. (2008). Mujeres, feminismos y género en España. Exit Book: revista semestral de Arte y Cultura visual, (9).

Tafur, Juan. (2005). La pasión de María Magdalena. España: Editorial Planeta.

Woolf, Virginia. (1992). Una habitación propia (traducción de Laura Pujol). Barcelona: Seix Barral. 